\title{
Intractable Abdominal Pain in a Patient With Spinal Cord Injury: A Case Report
}

\author{
Hye Kyung Park, MD, Dae Heon Song, MD, Young Moon Kim, MD, \\ Hong Geum Kim, MD, Soo Yeon Kim, MD, Myung Eun Chung, MD
}

Department of Rehabilitation Medicine, St. Paul's Hospital, The Catholic University of Korea College of Medicine, Seoul, Korea

Patients with spinal cord injury (SCI) may experience several types of chronic pains. Abdominal pain in patients with SCI has gained limited attention and little is yet known about its characteristics and mechanisms. It often has been regarded as visceral pain associated with constipation and distention. Neuropathic pains localized in the abdomen have rarely been reported. We experience a case of intractable abdominal pain in a patient with SCI, neither of visceral pathology nor of musculoskeletal origin. The nature of pain fulfilled the diagnostic criteria for neuropathic pains. The pain was therefore regarded as neuropathic and managed accordingly. The first- and second-line oral drugs available were being performed, unfortunately, adequate pain control was not achieved. We tried an intrathecal lidocaine injection as another treatment option, and the injection had considerable effects.

Keywords Abdominal pain, Neuropathic pain, Spinal cord injuries

\section{INTRODUCTION}

Chronic pain is found to be quite common in spinal cord injury (SCI) patients, with a prevalence ranging from $11 \%$ to $94 \%$ [1]. The presence of pain, especially severe pain, not only interferes with activities of daily living and effective rehabilitation, but also disrupts the psychological functioning and social integration of the person.

Chronic abdominal pain or discomfort is reported in

Received August 22, 2012; Accepted October 23, 2012

Corresponding author: Myung Eun Chung

Department of Rehabilitation Medicine, St. Paul's Hospital, The Catholic University of Korea College of Medicine, 180 Wangsan-ro, Dongdaemungu, Seoul 130-709, Korea

Tel: +82-10-3161-1012, Fax: +82-2-968-2307, E-mail: coltrane@catholic. ac.kr

(a) This is an open-access article distributed under the terms of the Creative Commons Attribution Non-Commercial License (http://creativecommons. org/licenses/by-nc/3.0) which permits unrestricted noncommercial use, distribution, and reproduction in any medium, provided the original work is properly cited.

Copyright ( $\odot 2013$ by Korean Academy of Rehabilitation Medicine about one-third of patients with long-term SCI [2]. However, abdominal pain in patients with SCI has only gained limited attention in research and little is known about its characteristics and mechanisms [2]. Abdominal pain is generally aggravated by constipation, urinary tract infection or food intake. It has therefore often been regarded as visceral pain [3]. When a visceral pathology cannot be identified despite careful and extensive evaluations, it has been suggested to classify the pain as neuropathic rather than visceral, and thus, treat it accordingly [4]. To the best of our knowledge, neuropathic pain localized in the abdomen alone has not yet been reported in Korea, while two cases with abdominal pains have been reported in Turkey [5].

We experienced a case of intractable neuropathic pain localized alone in the abdominal in a SCI patient, with neither visceral nor musculoskeletal pathology, who improved considerably when treated as neuropathic pain. We herein report our case with a review of the literature. 


\section{CASE REPORT}

A 37-year-old male patient was admitted to the department of rehabilitation medicine at our hospital with a major complaint for severe abdominal pains. He was affected by flaccid paraplegia in July 2011 due to a SCI at the T10 level in a traffic accident which led to compression fracture of vertebral body T10 with dislocation. After a long hospitalization in the intensive care unit, he developed a stage IV sacral pressure ulcer (length $10 \mathrm{~cm}$, width $10 \mathrm{~cm}$, depth $5 \mathrm{~cm}$ ) which was steadily worsened due to fecal incontinences despite the intensive dressings three times a day. Plastic surgeons, therefore, recommended a colostomy to provide a clean environment for healing the pressure ulcer. He also suffered from intermittent abdominal pain 1 month after the injury. The pain was mainly localized in the periumbilical area and did not appear in a radiating pattern. The initial pain scale was $60 \mathrm{~mm}$ on visual analog scale (VAS). Physical examination demonstrated no tenderness or distension to palpation of the abdomen. Peripheral blood tests showed elevated erythrocyte sedimentation rates with $69 \mathrm{~mm} / \mathrm{hr}$ and C-reactive protein was elevated to $3.77 \mathrm{mg} / \mathrm{dL}$. His past medical history included antibiotics therapy for acute cholecystitis a month ago. Computerized tomography (CT) on abdomen showed mild nonspecific gallbladder wall thickening (Fig. 1). Chronic cholecystitis was diagnosed and the patient was referred to the general surgery department for a colostomy and cholecystectomy. Laparoscopic cho-

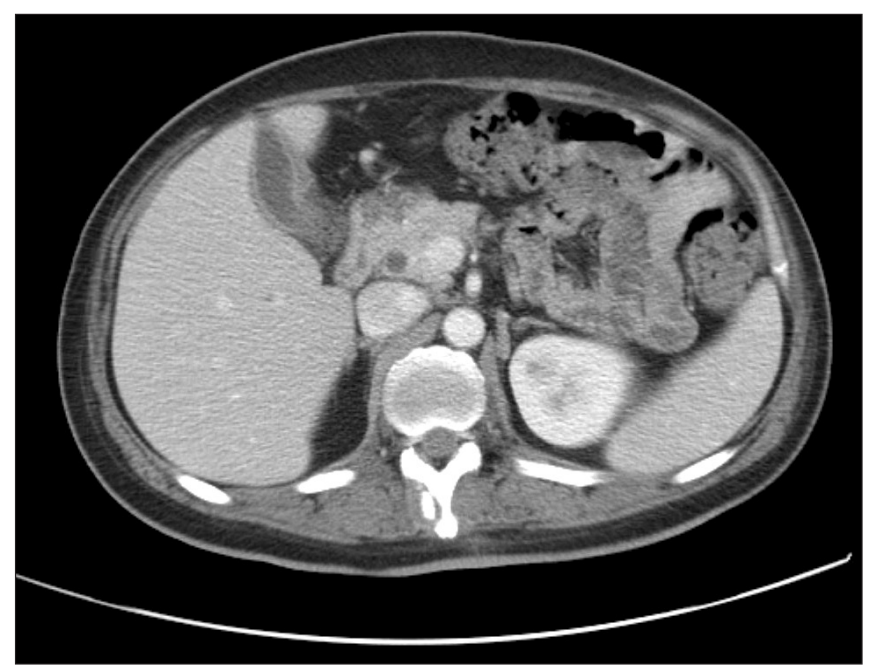

Fig. 1. Computerized tomography on abdomen showed a mild nonspecific gallbladder wall thickening. lecystectomy and sigmoid colon loop colostomy were performed on November 2011, but the abdominal pain around umbilicus persisted and became even more severe. The pain intensity scored $80 \mathrm{~mm}$ on VAS. The pain was not associated with food intakes or defecations. No other possible causes of visceral pain such as constipation, paralytic ileus or abdominal distension were identified. Laboratory findings on the peripheral blood tests were nonspecific and follow-up CT images of abdomen showed no abnormalities. The pain was not aggravated by positions, activities, movement and not associated with somatic tenderness, thus, we can rule out the possibility of musculoskeletal origin, such as mechanical instability, muscle spasm, overused syndromes. A magnetic resonance imaging scan of the thoracolumbar spine revealed no evidence for compression of the nerve root in the foramen by bone or disc that are correlated with the location of the pain. The nature of pain was either burning, pricking, electric shock-like, painful cold, tingling or an itching pain, which would fit the diagnostic criteria for neuropathic pain based on the Douleur Neuropathique 4 (DN4) questionnaire [1] and had a correlation with the dermatomal pattern within the level of injury. Thus, we concluded the pain as neuropathic at 5 months after the injury and exercised a medication according to guidelines [4]. We administrated amitriptyline $10 \mathrm{mg}$ twice a day, pregabalin $150 \mathrm{mg}$ four times a day, and oxycodone $5 \mathrm{mg}$ twice a day, after which the pain slightly subsided from 80 to $65 \mathrm{~mm}$ on VAS. However, he still suffered from insomnia and could not tolerate rehabilitation programs. He was then referred to the department of anesthesiology for intrathecal injections, with $12 \mathrm{~mL}$ of $0.4 \%$ lidocaine and $3 \mathrm{~mL}$ of $10 \mathrm{mg} / \mathrm{mL}$ triamcinolone. The pain level was reduced from 80 to $40 \mathrm{~mm}$ on VAS. The effect was not long-term and intrathecal lidocaine injection was scheduled more frequently, once a week for 3 consecutive weeks, keeping the oral medications. A repeated injection resulted in a remarkable decrease in pain level up to $30 \mathrm{~mm}$ on VAS for at least 2 months.

\section{DISCUSSION}

Patients with SCI may experience several types of chronic pain. The first step of approach to choosing the optimal treatment strategy for SCI pain is an understanding for the mechanisms and accurate classifications of the pain. The SCI Pain Task Force of the International As- 
sociation for the Study of Pain broadly classifies SCI pain into nociceptive and neuropathic pains [6].

A subtype of nociceptive pain, visceral pain is located either in the thorax, abdomen, or pelvis and is believed to be primarily generated in visceral structures. The reported prevalence of visceral abdominal pain varies from $5 \%$ to $38 \%$. Finnerup et al. [2] conducted a study to describe the characteristics of chronic abdominal pain in SCI patients. According to these authors, lower defecation frequency was more common in patients with abdominal pains, thus, suggesting that constipation plays an important role in abdominal pain following SCI [2]. Abdominal pain may be exacerbated by food consumptions and often improves with bowel movement or passing flatus which are both associated with distention [7].

In our patient's case, we can rule out acute abdominal pathologies, such as perforated viscus, cholecystitis (post cholecystectomy state), appendicitis, and bowel obstruction [8]. Also, the patient had a colostomy performed which provided a clean environment for optimal healing of pressure ulcer and causes of chronic visceral pains, such as constipation, paralytic ileus, abdominal distension were excluded. Abdominal pain in patients with $\mathrm{SCI}$ is sometimes considered neuropathic when visceral pathology cannot be identified despite the careful and extensive evaluations.

Approximately $30 \%$ of chronic pain in patients with SCI manifests as neuropathic. Two types of neuropathic pain appear to be specific to SCI. The first occurs in a dermatomal or segmental distribution at the level of injury, and is therefore, often referred to as at-level neuropathic pain. The second occurs in a more diffuse distribution below the level of neurological injury, and is therefore, referred to as below-level neuropathic pain [6]. Siddall [6] reported that at- and below-level accounted for $60 \%$ and $48 \%$ of neuropathic pain in SCI patients, respectively. In a study by Celik et al. [5] which evaluated the characteristics of neuropathic pain with SCI patients, the majority of patients reported symptoms in the lower extremities. Two of 50 patients reported periumbilical pain [5]. But neuropathic pain, which is localized only in the abdomen area, has rarely been reported in Korea or overseas.

In addition to accurate classification of the patient's pain, an understanding of the mechanisms for neuropathic pain is also important for proper treatment. Recently, animal and human studies have altered the properties of the nerve cells close to the site of SCI. These changes included increased responsiveness to peripheral stimulations and prolonged firing following a stimulus [6]. Further studies have demonstrated a number of changes in the following neurotransmitters and receptors that may lead to an increase in excitation or a reduction in inhibition which result in the change for firing properties of these spinal neurons: N-methyl-D-aspartate (NMDA), ion channels (including sodium and calcium channels), gamma-aminobutyric acid (GABA)ergic, opioid, serotonergic, and noradrenergic [6]. Recent treatment guidelines for neuropathic pain recommended tricyclic antidepressants, gabapentin, pregabalin, and mixed serotonin-noradrenaline reuptake inhibitors as first-line treatments and opioids (including tramadol) as secondline treatments [4].

In our patients, available first- and second-line drugs were administered according to guidelines, but unfortunately, adequate pain controls could not be achieved. Most studies indicated that the best available medications will only provide a $50 \%$ reduction of pain for an estimated one-third of people. If oral administration fails to provide adequate analgesia, local anesthetics may be helpful. We tried an intrathecal lidocaine injection and achieved a considerable effect. A few studies reported parenteral administration of the sodium channel blocker lidocaine to be effective for the treatment of neuropathic pain following SCI [9]. In another study, spinal anesthesia with subarachnoid lidocaine may also provide analgesia in SCI neuropathic pains [6]. Increased and abnormal sodium channels are known to be a source of neuronal hyperexcitability. A recent experimental study demonstrated expressions of abnormal sodium channels on dorsal horn neurons close to the spinal cord lesion, which was functionally linked to neuronal hyperexcitability and central pain behavior. Therefore, sodium channel blockers, such as lidocaine, can be tried as a pharmacologic option [9]. Combined intrathecal administration of morphine and clonidine was also found to produce a short-term reduction in pain for a group of people with neuropathic SCI pains [10]. However, local anesthetics administration is generally not as practical as an ongoing treatment, and thus, long-term administration remains a problem.

The limitation of our case is the lack of the long-term follow-ups for the duration of the effect. Further data on 
the long-term efficacy of intrathecal administration of lidocaine is needed.

In conclusion, we report a rare case of intractable neuropathic pain localized in the abdominal alone in a SCI patient. The management of intractable chronic pain is quite difficult, especially when the oral administration fails to provide adequate analgesia. In that case, an intrathecal injection of an agent with sodium channel-blocking properties or other local anesthetics may be other treatment options when considering the mechanisms behind the neuropathic pain.

\section{CONFLICT OF INTEREST}

No potential conflict of interest relevant to this article was reported.

\section{REFERENCES}

1. Calmels P, Mick G, Perrouin-Verbe B, Ventura M; SOFMER (French Society for Physical Medicine and Rehabilitation). Neuropathic pain in spinal cord injury: identification, classification, evaluation. Ann Phys Rehabil Med 2009;52:83-102.

2. Finnerup NB, Faaborg P, Krogh K, Jensen TS. Abdominal pain in long-term spinal cord injury. Spinal Cord 2008;46:198-203.

3. Ebert E. Gastrointestinal involvement in spinal cord injury: a clinical perspective. J Gastrointestin Liver Dis 2012;21:75-82.

4. Siddall PJ, Middleton JW. A proposed algorithm for the management of pain following spinal cord injury. Spinal Cord 2006;44:67-77.

5. Celik EC, Erhan B, Lakse E. The clinical characteristics of neuropathic pain in patients with spinal cord injury. Spinal Cord 2012;50:585-9.

6. Siddall PJ. Management of neuropathic pain following spinal cord injury: now and in the future. Spinal Cord 2009;47:352-9.

7. Stone JM, Nino-Murcia M, Wolfe VA, Perkash I. Chronic gastrointestinal problems in spinal cord injury patients: a prospective analysis. Am J Gastroenterol 1990;85:1114-9.

8. Neumayer LA, Bull DA, Mohr JD, Putnam CW. The acutely affected abdomen in paraplegic spinal cord injury patients. Ann Surg 1990;212:561-6.

9. Finnerup NB, Biering-Sorensen F, Johannesen IL, Terkelsen AJ, Juhl GI, Kristensen AD, et al. Intravenous lidocaine relieves spinal cord injury pain: a randomized controlled trial. Anesthesiology 2005;102:102330 .

10. Siddall PJ, Molloy AR, Walker S, Mather LE, Rutkowski SB, Cousins MJ. The efficacy of intrathecal morphine and clonidine in the treatment of pain after spinal cord injury. Anesth Analg 2000;91:1493-8. 